\title{
ECONOMÍA COLABORATIVA: DEFINICIONES Y ESCENARIOS
}

\section{COLLABORATIVE ECONOMY: DEFINITIONS AND SCENERIES}

\author{
Antonio Alaminos Chica \\ Universidad de Alicante (Spain) \\ alaminos@ua.es \\ Clemente Penalva Verdú \\ Universidad de Alicante (Spain) \\ clemente.penalva@ua.es
}

\section{Resumen}

En un contexto de acelerado cambio económico y social, mediatizado por la tecnología de la comunicación, la economía colaborativa es un fenómeno emergente especialmente significativo desde el punto de vista sociológico. Estas prácticas sociales, inicialmente orientadas a lo económico tienen importantes repercusiones en el conjunto del sistema social al generar nuevas relaciones sociales -y de poder- que atraviesan la totalidad de ámbitos de relación social: vida cotidiana y comunidad, participación ciudadana, gobernanza y marco jurídico, educación y cultura, relaciones laborales, etc.

El propósito de este artículo es, partiendo del diagnóstico ampliamente compartido sobre la indefinición de la economía colaborativa, establecer una primera sistematización conceptual que permita una clarificación de los fenómenos y procesos socioeconómicos involucrados. Tanto en sus aspectos relativos a contenidos, como al papel que juegan las formas de intercambio, combinando la mediación tecnológica con fórmulas tradicionales. A partir de dicha reflexión teórica se define un objeto de investigación que separa (si bien destacando la mutua influencia) - lo que se puede denominar "economía digital" en las que la organización de los procesos reproduce la estructura de mercado tradicional, de aquellas otras experiencias de base solidaria, naturaleza 
comunitaria y orientación resiliente que forma la "economía colaborativa". Posteriormente, considerando diferentes escenarios de futuro que ponen en el centro la nueva configuración de la economía global, se indaga sobre las posibilidades que un programa de investigación que sea capaz de describir y medir el alcance y las formas de las iniciativas de innovación social orientadas a la economía y su derivación sociopolítica.

\title{
Palabras clave
}

Economía colaborativa, economía digital, prácticas sociales, resiliencia.

\begin{abstract}
In a context of accelerated economic and social change, mediated by communication technology, the collaborative economy is an emerging phenomenon that is particularly significant from a sociological point of view. These social practices, initially oriented to the economic, have important repercussions in the social system as a whole, generating new social relationships -and power- that go through all areas of social relationship: daily life and community, citizen participation, governance and framework legal, education and culture, labor relations, etc. The purpose of this article is, starting from the widely shared diagnosis about the lack of definition of the collaborative economy, to establish a first conceptual systematization that allows a clarification of the phenomena and socioeconomic processes involved. Both in its aspects related to the contents, as well as the role played by the forms of exchange, together with the combination of technologies with traditional formulas. From this theoretical reflection, a research object is defined that separates (although highlighting the mutual influence) - what can be called "digital economy" in which the organization of processes reproduces the traditional market structure, of those other experiences of solidarity, community nature and resilient orientation that forms the "collaborative economy". Subsequently, considering different future scenarios that place the new configuration of the global economy in the center, it is reflected on a research program that is able to describe and measure the scope and forms of social innovation initiatives oriented to the economy and its sociopolitical consequences.
\end{abstract}

\section{Key words}

Collaborative economy, digital economy, social practices, resilience. 


\section{Extended abstract}

It is evident by virtue of the proposals considered, and currently in debate, that the confusion over the collaborative economy, its purpose and dependence on technological developments, are allowing an effective tool of social transformation to be buried in defining ambiguities. This situation is aggravated by being absorbed in the commercial dynamics of consumer capitalism, where, as happened in its time with environmentalism and other alternatives, the notion of "collaborative" becomes an attribute that characterizes the products, retaining all the logic of the generation of surplus value. In that sense, the analysis of these emerging processes is an academic but also socially significant task.

It will be the knowledge of the authentic nature that defines the relationships inserted in these dynamics of social, economic and symbolic exchange, which will allow first an adequate measurement and then a possibility of follow-up and evolution of change. Both in terms of social groups, ideological alternatives, and social relationships.

The proposal of Matties and Närhi (2016) constitutes a relevant starting point to which, obviously, the social and ideological dynamics that make up real alternatives of "collaborative economy" should be included. The qualitative leap between the scenarios of "local resilience" to "global commons", thanks to the organizational structure facilitated by the new communication and information technologies; define the great operational challenge, but also the theoretical challenge facing alternative visions to globalized consumer and financial capitalism.

\section{INTRODUCCIÓN}

El concepto de prácticas sociales expresa los comportamientos recurrentes que una determinada sociedad considera como válidos y que son compartidos de manera más o menos generalizada. Para la exposición de esta investigación teórica, vamos a considerar especialmente las que están orientadas al comportamiento económico. Particularmente, en la medida que da forma y densidad a una cultura específica que da legitimidad a dichas prácticas. Este sustrato cultural es la base a partir de la cual las prácticas se transforman y reemplazan a través del tiempo, difiriendo de una sociedad a otra. En un contexto de extensión acelerada de la economía, mediada y diversificada por la tecnología de la comunicación, se asiste, principalmente a partir de la crisis financiera de finales de la década pasada, a un conjunto de prácticas económicas que rescatan el principio de cooperación y comunidad para establecer un intercambio de productos y servicios entre iguales. Del mismo modo que las tecnologías han 
servido de acelerante de las dinámicas económicas capitalistas, ofrecen una posibilidad para que economías alternativas sean viables a escalas que no eran factibles con anterioridad.

En los últimos tiempos se observan un conjunto de prácticas económicas basadas en el intercambio cooperativo que ha llamado la atención como un claro ejemplo de innovación social por parte de los científicos sociales de diferentes disciplinas: sociología, economía, marketing, antropología y estudiosos de las organizaciones. Al conjunto de estas prácticas económicas que se entienden como nuevas y vinculadas a las tecnologías de la comunicación, se les ha denominado de diferentes formas, hecho que denota la falta de consenso en su definición.

Uno de los términos más utilizados, como muestra su espectacular incremento desde 2015 en las búsquedas de Google Trend (Raffini, 2016: 130) es el de "economía colaborativa", como traducción de "sharing economy". Se podría decir que la expresión "economía colaborativa" se ha incorporado al lenguaje cotidiano y ocupa con cierta frecuencia, espacios informativos y de divulgación en los medios convencionales, algunos de los cuales no sólo describen algunas de sus prácticas, sino también señalan los debates sociales y académicos asociados. El debate se establece en cuanto a la discusión sobre su contribución a un nuevo modelo económico o al incremento real de las capacidades y autonomía de las sociedades (Alonso, 2017; Laín, 2017).

La definición de economía colaborativa deviene imprescindible porque el ejercicio de establecer los límites en cuanto a las prácticas económicas, por lo que se refiere a contenido (qué se intercambia), forma (cómo se intercambia), motivación y existencia de mediación tecnológica; nos puede ofrecer las bases para describir (en sus dimensiones e indicadores orientados a la medición), comprender y explicar las mutuas relaciones (incluso entre las propias prácticas económicas) que se producen en torno a un fenómeno que está en continuo crecimiento, y que aparenta ser sostenido e irreversible.

Esta economía colaborativa, forma parte de la "economía digital" (las relaciones de intercambio monetarizadas orientadas a la producción y distribución de bienes y servicios que se efectúan a partir de plataformas en internet). Los datos de su extensión no pueden ser más elocuentes. En un informe de la CNMC (2016) se muestra el espectacular incremento de las inversiones en iniciativas a nivel mundial relacionadas con la economía colaborativa desde 2013. Por otro lado, los datos de la Unión Europea señalaban en 2016 que más de la mitad de los ciudadanos europeos conocen la economía colaborativa, que una de cada 6 personas la usa y que más del $5 \%$ de la población de la UE ya ofrecía productos y servicios a través de estas plataformas (Union Europea, 2016). 
Figura 1. Evolución de la inversión mundial anual en plataformas de economía colaborativa

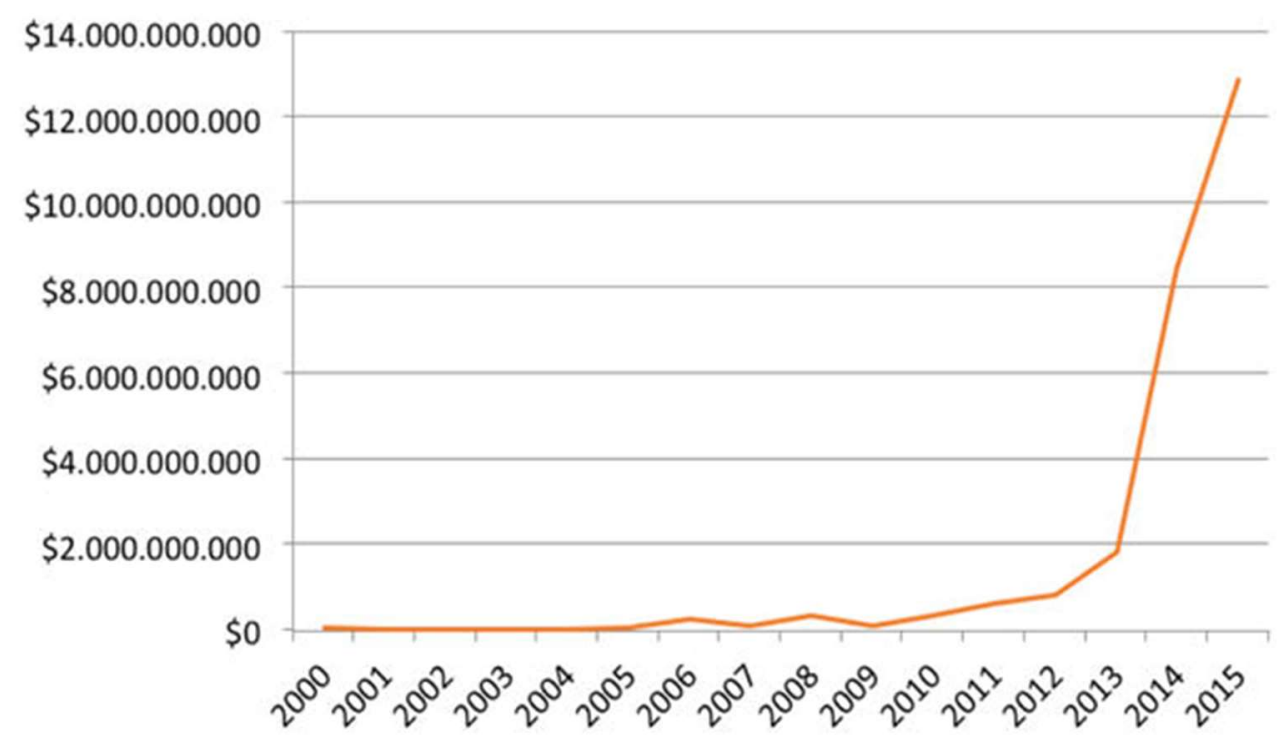

Fuente: CNMC $(2016,21)$

Pero esto se refiere únicamente a la parte más visible y registrada, puesto que las prácticas económicas colaborativas suponen (igual que un conjunto de actividades que tradicionalmente no son visibilizadas, contabilizadas y reconocidas como es el trabajo doméstico, el de cuidados, etc.) mucho más que lo que este gráfico refleja. Un mucho más que escapa de su registro, imprescindible para la contabilidad nacional y susceptible de ser fiscalizado. Esta cuestión (el registro) es muy importante para la contabilización económica formal, pero en su tratamiento sociológico se corre el riesgo de considerar economía colaborativa únicamente lo que se mueve en la economía oficial (formal). Como decíamos anteriormente, definir qué es la economía colaborativa no es fácil. Su conceptualización se puede realizar estableciendo los límites de estas prácticas y su discusión en cuanto alcance, consecuencias en torno a sus efectos en el ámbito socioeconómico, a los ámbitos sociales que afectan (red social, empleo, satisfacción de necesidades, modelo económico, e incluso comportamiento político), así como los debates asociados a sus regulaciones administrativas y políticas públicas.

En un plano institucional, la Comisión Europea, en su estrategia para el desarrollo de un mercado único, a la hora de establecer una "agenda para la economía colaborativa" que active el empleo y para usarla como "orientación jurídica y política a las autoridades públicas, los operadores del mercado y los 
ciudadanos interesados"; la define, atendiendo a su carácter mercantil, digital, jurídico e identificando a los agentes del intercambio, de esta manera:

“A los efectos de la presente comunicación, el término "economía colaborativa" se refiere a modelos de negocio en los que se facilitan actividades mediante plataformas colaborativas que crean un mercado abierto para el uso temporal de mercancías o servicios ofrecidos a menudo por particulares. La economía colaborativa implica a tres categorías de agentes i) prestadores de servicios que comparten activos, recursos, tiempo y/o competencias - pueden ser particulares que ofrecen servicios de manera ocasional ("pares") o prestadores de servicios que actúen a título profesional ("prestadores de servicios profesionales"); ii) usarios de dichos servicios; y iii) intermediarios que - a través de una plataforma en línea - conectan a los prestadores con los usuarios y facilitan las transacciones entre ellos" ("plataformas colaborativas"). Por lo general, las transacciones de la economía colaborativa no implican un cambio de propiedad y pueden realizarse con o sin ánimo de lucro." (Comisión Europea, 2016)

Al margen de esta definición institucional, muy útil para contextualizar un marco que oriente la investigación sobre su naturaleza, estructura y agentes implicados en el ámbito europeo, un buen inicio es describir qué actividades se llevan a cabo, qué es lo que se hace dentro de este marco de la economía colaborativa. Bostman y Roger (2010) utilizan el término "consumo colaborativo" para indicar las actividades de trocar (bartering), prestar (lending), donar (giving), alquilar (renting) e intercambiar (swapping). Todas estas actividades se englobarían en tres categorías: acceder a productos o servicios sin necesidad de tener la propiedad de los bienes ("product servcie system"); mercados de redistribución ("redistribution markets") de bienes que alargan su vida después de haber sido usados (segunda mano, reutilización, reciclado, reparación) y estilos de vida colaborativos ("collaborative lifestyles") que supone el intercambio de bienes no tangibles (espacio, tiempo, habilidades o dinero). Otras clasificaciones tienen que ver con las prácticas de: recircular bienes (eBay); incrementar la utilización de bienes duraderos (por ejemplo, Zipcar, Relay Rides, Uber, CouchSurfing, Airbnb); intercambio de servicios (bancos de tiempo); de compartir bienes (Mama Bake, Soup Sharing, and EatWithMe) (Codagnone y Martens, 2016). Un rasgo clave que explica las dificultades de su clasificación es la confusión entre actividades de producción y consumo, por el hecho de que los roles no son asimétricos, en el sentido de que son jugados al mismo tiempo por los participantes en la colaboración. 
Es el factor tecnológico el que permite esta conjugación de roles y es el que se utiliza para la denominación y descripción de este conjunto de actividades. Así, tenemos los términos que la incluyen como una continuación o nuevo desarrollo de la anteriormente denominada "economía digital" (OCDE, 2018) con el carácter de interacción entre pares (peer to peer; o P2P) gracias a las plataformas comerciales que permiten los intercambios. En estas plataformas, el usuario recibe y envía objetos y servicios, además de información sobre las características del objeto de intercambio y sobre los mismos usuarios, en secciones específicas que sirven para valorar online la satisfacción del intercambio.

La perspectiva focalizada en los agentes implicados y la confusión de roles jugados en el intercambio nos remite a la idea de prosumidor. Se trata de un prosumidor "ampliado" que sobrepasa las fronteras del diseño o el uso de productos de consumo, para convertirse en proveedor y facilitador de servicios compartidos. El individuo es prosumidor de bienes y servicios, pero también de información, en un ciclo continuo que retroalimenta la red registrando la relación en términos de valoración del intercambio y de reputación de los agentes. Dos aspectos básicos se han de incluir para completar la descripción de la red: no es necesaria la mediación del dinero (al menos del dinero oficial), la propiedad del bien pierde centralidad y se valora más la posibilidad del acceso al mismo. Todas estas transacciones se realizan en un entorno donde, supuestamente, las relaciones se establecen entre iguales, sin intermediación, lo que da pie a cierta idea de comunidad. Una idea de "comunidad" que está en el origen de muchas iniciativas altruistas ampliamente conocidas y extendidas (pensemos en el software libre, el creative commons o el conocimiento libre), pero no de todas.

La actividad mercantil está muy presente en esta economía colaborativa, marcada por la búsqueda de beneficio, pero en muchos casos se le añaden otros valores, además de los meramente utilitarios y monetarios. Aunque estos valores acompañan a la retórica publicitaria de las grandes plataformas que actúan transnacionalmente, no dejan de estar presentes, y existen un conjunto de investigaciones que indagan hasta qué punto son elementos motivadores a la hora de usarlas, estableciendo el contraste entre actitudes positivas y participación (Hamari, Sjöklint y Ukkonen, 2015) y los determinantes no racionales y monetarios en la satisfacción del consumo colaborativo (Möhlmann, 2015). También se han estudiado las motivaciones medioambientales, sociales y económicas según grupos sociodemográficos y tipo de participante (usuario o provedor) (Böcker y Meelen, 2017). Así, se añaden a las motivaciones utilitarias individuales (acceso fácil, precio bajo, calidad contrastable, ingresos monetarios extralaborales o empleo informal, aprovechamiento del valor de uso prolongado de los productos no utilizados); otras de carácter menos utilitario y más colectiva como pueden ser la sociabilidad (estrechar los lazos de una red preexistente o crear una nueva), la protección del medio ambiente (gracias a la recirculación de 
bienes), y la afirmación ideológica (posibilidad de implicarse en un proyecto alternativo al mercado convencional).

El estudio de la economía colaborativa que se ha producido en estos últimos años la define, por tanto, en tres elementos que parecen inseparables:

a) Actividades donde la propiedad pierde peso con respecto al uso, incluyendo la posibilidad de compartir sin mediación del dinero

b) La idea de posibilidad de negocio (aunque no sea absolutamente necesario) que incluso pueda atravesar las fronteras estatales y formar grandes compañías de enorme peso en la economía mundial, y

c) La presencia de plataformas tecnológicas que facilitan las transacciones e intercambios.

Observando estas categorías, el auge y difusión de la economía colaborativa está mostrando que podría llegar a incluir todo lo que está presente en el mercado para la satisfacción de necesidades: bienes de consumo -alimentos, vivienda y hospedaje, herramientas, medios de locomoción, mobiliario, electrodomésticoshabilidades y servicios - profesionales o no profesionales-, conocimiento, incluso dinero. Si lo observamos desde un punto de vista sustantivo de la economía (como aquella esfera de lo social en el que las sociedades organizan la producción de bienes y servicios para la satisfacción de necesidades, y de la cual se derivan relaciones e intercambios en los cuales lo utilitario e instrumental como motivación de la acción puede ser secundario) su enorme despliegue puede hacer ver a la gente de la enorme potencialidad de la colaboración, y que esta es posible sin la mediación del dinero o sin beneficio lucrativo.

Para los objetivos de este trabajo y como delineamiento de investigación, la economía colaborativa es aquella que cumple con el criterio de englobar un conjunto de prácticas económicas que no persiguen el beneficio económico y que no son proporcionadas por el sector público, independientemente de que medie o no lo digital. Desde un planteamiento parsimonioso, empleamos aquí una definición operativa y sociológica que trasciende lo digital para centrarse en lo social. Con esto queremos resaltar la confusión existente -nada inocente, con un trasfondo ideológico que sirve para definir en cuanto a estrategias de marketing lo que no es por lo que es- entre la economía colaborativa y la economía digital. Si atendemos a sus definiciones clásicas y no institucionales, lo colaborativo es una parte esencial de la economía, en la cual sólo una parte es monetarizada y orientada al lucro. Es decir, lo que se nombra alude a lo que no es, que es lo que hace la definición institucional de la economía colaborativa. 
Desde nuestra perspectiva, a expensas de delimitar cuantitativamente su tamaño, nos estamos refiriendo a una esfera de lo económico que se caracteriza porque no persigue el lucro y en el interior del cual sólo una parte sería digital: aquel espacio de prácticas económicas resultado de la intersección entre lo no lucrativo y digital.

Figura 2. Esferas de la economía colaborativa atendiendo a su mediación tecnológica digital

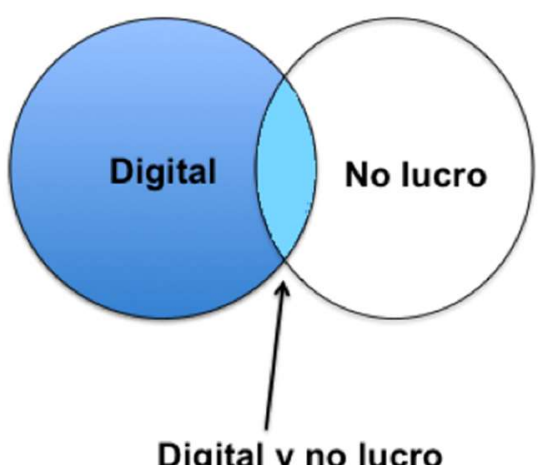

Fuente: elaboración propia

El estudio de las formas de intercambio es interesante porque permite observar en su conjunto el verdadero carácter de esta colaboración. Una colaboración que se diferencia de las que han existido a lo largo de la historia de las sociedades humanas en que se incorpora la tecnología en red. Desde la antropología, se recuperan los conceptos de intercambio (regalo, cuidado -mothering- $y$ reciprocidad) para, entre otras cosas, observar las diferencias a la hora de clasificar las prácticas entre sharing in y sharing out (Belk, 2014). La diferencia es que, aunque las dos incluyen intercambios, la segunda no lleva incorporado ningún sentido de pertenencia a una comunidad y se reserva para los intercambios hacia fuera de la misma. Esta distinción es útil para observar aquellas prácticas que derivan en situaciones más mercantilistas y con menor sentido de lo común. El anonimato, las razones exclusivamente instrumentales y el objetivo de lucro permite discriminar el tipo de colaboración que establece la red. Las secciones de las plataformas diseñadas para la valoración de los usuarios anónimos es el mejor indicador de su carácter no comunitario. 
Estos son los parámetros entre los que se ha de mover la discusión sobre este fenómeno actual de la economía colaborativa y su orientación a la acción colectiva y participativa. El debate no únicamente tiene que ver sobre su integración en el mercado y la reproducción de las prácticas mercantilistas insertas en las configuraciones económicas marcadas por el neoliberalismo económico (utilitarismo, acumulación, desregulación del trabajo); sino también por sus "falsos" efectos beneficiosos medioambientales o sociales. Estas plataformas no dejan de fomentar el consumismo (la reutilización no compensa los costes ambientales de la mayor extensión y acceso al consumo), la turistificación y gentrificación de las ciudades, y el daño al comercio local y a otras actividades profesionales (Slee, 2016).

La economía colaborativa se observa como uno de los fenómenos que ejemplifican la nueva configuración de las sociedades en estos momentos de cambio social. Aunque sólo sea potencialmente, apuntan a una nueva reinserción de lo económico en lo social. Apoyada sustancialmente por el empleo de las tecnologías de la comunicación en red, nos ofrece una oportunidad para pensar (a pesar de las críticas por su deriva mercantilista y desreguladora del trabajo, principalmente) en un "común" que vuelva a insertar las relaciones y prácticas económicas en lo social.

La economía, que fue apartada de lo social y dividida en dos esferas, la colectiva y la individual, identificadas, respectivamente, en los ámbitos del Estado (la autoridad normativa de las relaciones económicas) y el del mercado (regulador de las relaciones espontáneas de los agentes económicos individuales que eligen racionalmente su comportamiento económico), queda incluida en lo social bajo una forma nueva, para reinsertar la economía en la sociedad (Pais y Provasi, 2015).

Desde el punto de vista teórico, esta inclusión permite recuperar algunos de los fundamentos de la sociología económica (Polanyi, Granovetter) que aprovecha los aportes de la antropología del intercambio económico para negar el principio de que la circulación de bienes y servicios está fundamentada únicamente en razones instrumentales, o para hacer notar que la economía no se define únicamente por el mercado, sino como la forma en que las personas solucionan necesidades. La economía colaborativa permite observar cómo entre la redistribución (autoridad del Estado) y el intercambio (basado en el mercado), emerge la reciprocidad, cuyo principio rector serían las relaciones sociales horizontales.

En ese sentido, la economía colaborativa se situaría entre las relaciones anónimas del mercado y las relaciones jerárquicas del Estado. Es decir, entre los dos modelos de política económica que en las pasadas décadas han regido: el 
intervencionismo de la economía (keynesianismo y Welfare State) y el neoliberalismo. La economía colaborativa digital, aunque augura una "nueva economía" por su espectacular crecimiento en los últimos años y por los nuevos desarrollos y extensión de su forma "tecnológica", no es del todo "disruptiva". Desde los inicios de la crisis del Estado de Bienestar (que es anterior a la presente y aguda crisis económica) se habla del Tercer Sector, notando cómo ciertas concepciones y aspectos de lo que es la economía colaborativa son "hijos" de la fusión liberal y socialdemócrata de la Tercera Vía. Su desarrollo ha conducido, en muchos casos, a la aportación del trabajo voluntario y a la implicación de la sociedad civil con el fin de reducir los gastos públicos destinados a servicios, con una reducción de los salarios y de la calidad de los servicios (Pirni y Raffini, 2015).

Desde esta perspectiva analítica, como hipótesis, la economía colaborativa, en sus aspectos "ideales" (exagerados en el marketing de las grandes corporaciones que utilizan la economía colaborativa como etiqueta), podría derivar hacia alguno de los dos modelos conocidos (mercado y Estado). El primero es empírico (la economía colaborativa ya está sirviendo para desregular trabajo y comercio, y para acumular capital), el segundo es teórico (si considera estas actividades como beneficiosas para el bienestar general, dotándolas de reconocimiento jurídico a nivel local, fomentándolas en los niveles verdaderamente comunitarios y en las prácticas no lucrativas), como alude Santos a partir del concepto de "Estado experimental", fijándose en iniciativas gubernamentales iniciadas en países latinoamericanos (Santos, 2014). Se observa, no obstante, cómo las autoridades (desde la UE a los gobiernos locales) definen la economía colaborativa como un nuevo campo donde activar la actividad productiva, el consumo y el mercado de trabajo desde la base de lo local ${ }^{1}$, pero no se dispone de tantos datos sobre el efecto económico de estas políticas. Resulta obligado resaltar que este interés institucional en la economía colaborativa no obedece únicamente a la constatación del incremento de la mediación digital en la economía, sino también al observarla como una de las salidas a la crisis actual porque puede estimular el crecimiento económico y el empleo. Se trata de una salida desde abajo, en el sentido que el Estado ha perdido su papel de impulsor directo de la economía mediante políticas expansivas y de protector social. En estos momentos de crisis, el Estado ha actuado en un sentido totalmente contrario. Las políticas de austeridad aplicadas por parte de todos los gobiernos europeos han reducido el papel del Estado como agente capaz de paliar los efectos de la depresión económica sobre los sectores más afectados.

1 Ver portal sobre Collaborative Economy de la Comisión Europea:

http://ec.europa.eu/growth/single-market/services/collaborative-economy_en) 


\section{LA CRISIS Y LA RESILIENCIA SOCIAL}

Las sociedades reaccionan de diferentes maneras para cubrir la renuncia de las instituciones estatales. En parte, esas reacciones tienen que ver con aspectos como son la cultura cívica y organizativa, los valores de referencia solidarios, la estructuración comunitaria o societaria. En dichos contextos de referencia, las crisis económicas juegan un papel de "estresor" en las sociedades. Tras un tiempo más o menos prolongado de situación de shock sobre la sociedad civil, producida precisamente por la abrupta caída desde el cénit del ciclo expansivo de la economía a la depresión, la sociedad ha ido innovando -o "renovando"diferentes estrategias de adaptación al nuevo contexto. Como se ha definido en otro lado (Alaminos, Penalva y Domenech, 2014), la resiliencia es un proceso dinámico que tiene como efecto la adaptación positiva en contextos de gran adversidad, superándola. La resiliencia es esta capacidad de resistencia, adaptabilidad y superación de las sociedades a conservar su forma tras haber sido afectadas por malas experiencias. La resiliencia tiene un sustrato sociocultural, remite a experiencias y prácticas observadas como exitosas por parte del cuerpo social y que son actualizadas ante momentos de crisis social. La resiliencia, en su aplicación en las ciencias naturales, hace referencia a la capacidad que tiene un cuerpo deformado para recuperar su forma original, y tiene un componente relacionado con la capacidad de memoria de un material para recuperarse. Desde esta perspectiva, Magrinyà y Balanzó (2015), por ejemplo, hablan de la importancia de la memoria social (que se remonta a los años 30 del siglo pasado) en las experiencias de economía cooperativa y de autogestión actuales en los centros sociales de Barcelona, conectando los ciclos de renovación adaptativa con la evolución urbana de esta ciudad.

Muchas de las actividades resilientes son estrategias individuales que derivan en prácticas económicas de base, en muchos casos, comunitaria, donde la red de apoyo y el capital social son conceptos esenciales para la comprensión de su fundamento y extensión. Los hechos que apoyan estas afirmaciones provienen, en primer lugar, como se ha señalado más arriba, por la retirada de la protección del Estado y la derivación al Tercer Sector de muchas responsabilidades en cuanto a derechos y garantías. En segundo lugar, por las iniciativas individuales o familiares observadas para paliar los efectos de la crisis. En contextos de crisis se buscan alternativas para incrementar los ingresos y disminuir los gastos, como pueden ser practicando el intercambio de servicios o evitando el dinero (Unión Europea, 2014). Estas reacciones, que surgen como respuestas a crisis coyunturales, en la media que demuestren sus ventajas o beneficios, pueden convertirse en estructurales, integrando y definiendo nuevas prácticas sociales. En tercer lugar, se ha producido una extensión de las ideas y concepciones sobre la vida económica que incluye elementos valorativos del intercambio. Unos criterios valorativos que van más allá de la obtención de beneficio monetario: 
obtener un valor social -cohesión, integración, formación, bienestar, felicidad...en equilibrio con el medio ambiente y mediante la utilización de recursos locales. En estas redefiniciones de lo económico se incluye también la idea de un consumo responsable, con concepciones más sofisticadas sobre estilos de vida, modelos alternativos de intercambio económico e incluso societales: decrecimiento, buen vivir, son ejemplos de ello. Algunas de estas ideas y motivaciones toman impulso al situar a las entidades financieras en el centro de las críticas, responsabilizándolas -junto con una clase política sumisa a éstas- del origen de la crisis (Alaminos y Penalva, 2013).

Desde este enfoque (prácticas económicas locales, autogestionadas, sin finalidad de lucro, comunitarias y resilientes), un estudio pormenorizado de la economía colaborativa necesita un índice que exprese el repertorio de prácticas económicas que se establecen en un territorio, así como el grado de conexión entre ellas y de conexión intergrupal. Una manera de clasificarlas, independientemente del contenido del intercambio, es insertarlas en un espacio relacional estructurado según dos ejes relativos a la naturaleza de los vínculos sociales de los agentes implicados y la finalidad de la ayuda cooperativa. En el Gráfico 2 se puede representar los polos entre los que se mueve, por un lado, el grado del vínculo social (del anonimato de las donaciones a "bancos de alimentos" a la confianza en las "monedas sociales"); y, por otro, la orientación sociopolítica e ideológica que guía estas prácticas (desde el regreso a las situaciones de bienestar previas a la crisis hasta el cuestionamiento del sistema).

En ese sentido, la economía colaborativa, gracias a las nuevas tecnologías, adquiere la potencialidad de cambiar de nivel de actuación, pasando de ser actividades fragmentadas de supervivencia a alcanzar una mayor complejidad y estructura. Al mismo tiempo, su expansión por las redes de comunicación permite un salto cualitativo desde la resistencia y la resiliencia a convertirse en un auténtico vector de cambio y transformación. La potencialidad aumentada de coordinación, vinculada con contenidos de solidaridad tiene, de encontrar las expresiones adecuadas, una capacidad de alcanzar impactos elevados.

Existen otras aproximaciones para clasificar las actividades, como es la que ofrece Zubero (2015). Su clasificación se expresa a partir de una serie de círculos concéntricos donde el núcleo representa la economía de mercado y el externo las formas de intercambio alternativas más opuestas a las mercantilistas. Todas las representaciones tienen sus ventajas o sus desventajas y es, en definitiva, el investigador quien debe decidir cuál de ellas le ofrece más oportunidades analíticas y de generación de hipótesis. 
Figura 3. Estructura y dinámica de las prácticas económicas de resiliencia social

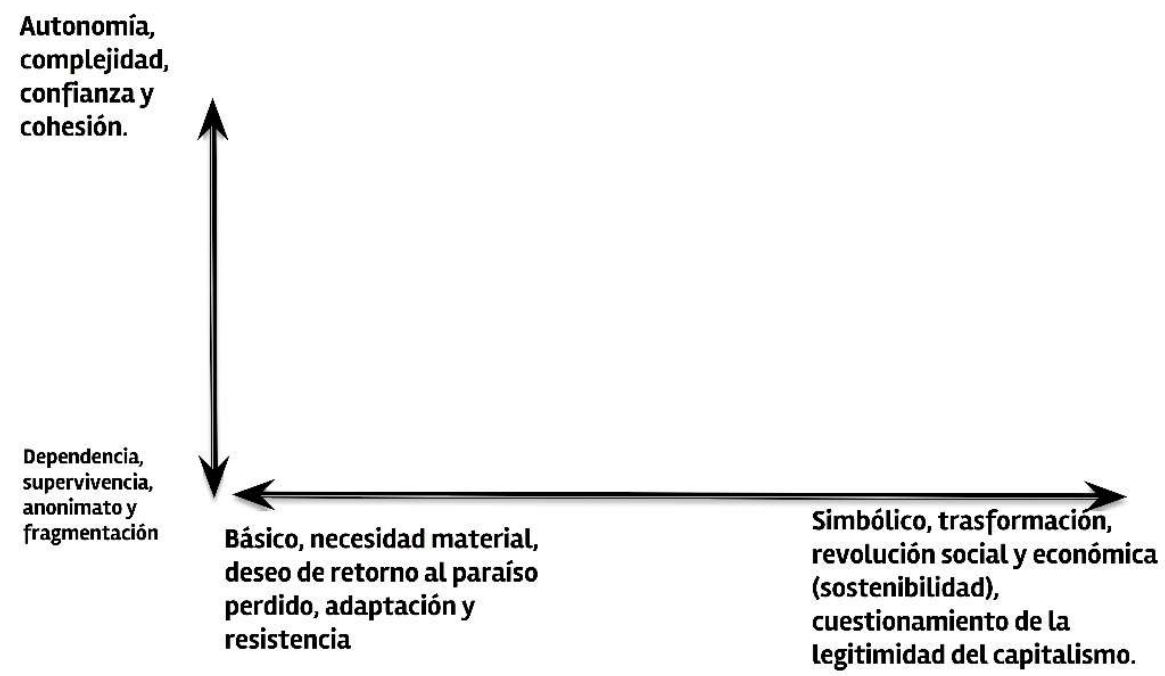

Fuente: Alaminos, Penalva y Domenech (2014)

Para establecer los parámetros donde ubicar las prácticas económicas resilientes ofrecemos el siguiente cuadro, en el cual se representan en oposición lo que define la "economía colaborativa" frente a la "economía digital"; y asumiendo, como explicábamos al inicio de este artículo que una parte de la economía colaborativa, entendida en términos comunitarios, también puede ser mediatizada por las tecnologías de la comunicación digital.

No obstante, como hipótesis de trabajo, consideramos que existe una relación contradictoria entre ambas esferas de la economía actual, en el sentido de que las consecuencias de la extensión de la economía digital son un deterioro de las condiciones de vida sociales. La precariedad y el subempleo se están instalando en las economías occidentales provocando un incremento de la desigualdad y la pobreza que son consecuencia directa de los nuevos mercados digitales. De igual modo, siguiendo el argumento, las prácticas resilientes forman el contrapunto de resistencia y supervivencia a esta situación sobrevenida por la economía digital. Es una forma alternativa que, precisamente, se alimenta de las consecuencias sociales del auge de la economía digital. Por otro lado, las prácticas de consumo y la bondad del intercambio colaborativo a las que apelan las campañas publicitarias de las plataformas no dejan de sugerir el potencial autorganizativo de las redes y sus agentes. Por ejemplo, las redes surgidas de los propios usuarios. Por ejemplo, redes que se forman a partir de la regularidad de las coincidencias en los itinerarios de los usuarios de Blablacar. 
Figura 4. Economía digital vs economía colaborativa

\begin{tabular}{|l|l|}
\hline Economía digital & Economía colaborativa \\
\hline Lucro & No lucro \\
\hline No comunitaria & Comunitaria \\
\hline Confianza digital (anónima) & Confianza interpersonal \\
\hline Relaciones anónimas & Relaciones personales \\
\hline Dinero & $\begin{array}{l}\text { Moneda (social). intercambio de horas, } \\
\text { servicios, productos }\end{array}$ \\
\hline Valor de cambio & Valor social \\
\hline Relaciones verticales (plataformas privadas) & $\begin{array}{l}\text { Relaciones } \\
\text { sociales) }\end{array}$ \\
\hline Intercambio & Reciprocidad \\
\hline Precariedad y exclusión & Resiliencia e integración \\
\hline Neoliberalismo & Comunismo \\
\hline
\end{tabular}

Fuente: elaboración propia

Por otro lado, el trabajo precario que proponen estas plataformas, da pie a la organización de sus trabajadores para reivindicar la mejora de sus condiciones de trabajo y de su relación laboral con la empresa. Es decir, ser reconocidos como trabajadores dependientes y no como trabajadores autónomos. En ocasiones, se han formado cooperativas de extrabajadores de las plataformas que ofrecen los mismos servicios que ofrecía su antigua empresa ${ }^{2}$. En términos de pregunta de investigación, el reto sería -tras las definiciones y clasificaciones pertinentes de las prácticas colaborativas- establecer las medidas de esta esfera resiliente de la economía para efectuar posteriormente su cuantificación.

2 https://www.eldiario.es/desde-mi-bici/Pajara-Ciclomensajeria-alternativa-DeliverooMadrid_6_825977398.html; http://www.eldiario.es/catalunya/trabajo/repartidores-Deliverooconvocan-primera-economia_0_658985300.html 


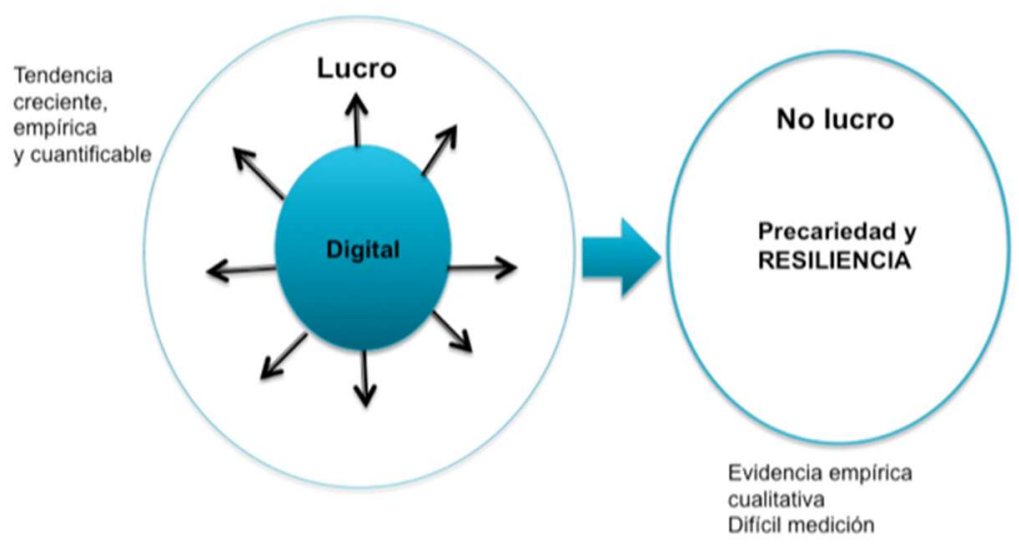

Fuente: elaboración propia

\section{ESCENARIOS}

Así, en un contexto generalizado de innovación tecnológica, con sustanciales cambios en las relaciones de consumo laborales, económicas y sociales, se entrecruza la innovación social. El tejido de las redes tecnológicas de la comunicación queda atravesado por las redes sociales que promueven el incremento del valor social frente al valor monetario. En estas dinámicas de cambio, las innovaciones sociales serían aquellas innovaciones que son sociales tanto en sus fines como en sus medios. Hacen referencia a nuevas ideas (productos, servicios y modelos) que simultáneamente satisfacen de modo más eficaz las necesidades sociales y crean nuevos modos de relación social. Esto es, son innovaciones que no sólo generan valor para la sociedad en su conjunto, sino que también incrementan la capacidad de acción de toda la sociedad. Esta definición, que proviene del Bureau of European Policy Advisers, es recogida por Mozón y Chavez (2018) para hacer énfasis en su carácter sistémico, en el sentido de transformación hacia modelos de sociedad más participativos. Consideramos, desde esta perspectiva, la interesante aportación que introduce la economía de los bienes comunes. Matties y Närhi (2016: 80), a partir de Bauwens y Lievens (2013), muestran cuatro escenarios futuros globales que describen esta mutua interacción entre redes tecnológicas y redes sociales. Se muestran relacionados cuatro escenarios que son producto, por un lado, del carácter de las redes interconectadas entorno a los polos centralización / descentralización de la información y la toma de decisiones y, por otro, de la naturaleza de las redes en 
cuanto al principio que regulan las mismas, en un eje formado por los polos de orientación al beneficio económico/beneficio social.

Figura 6. Escenarios futuros globales del cambio digital

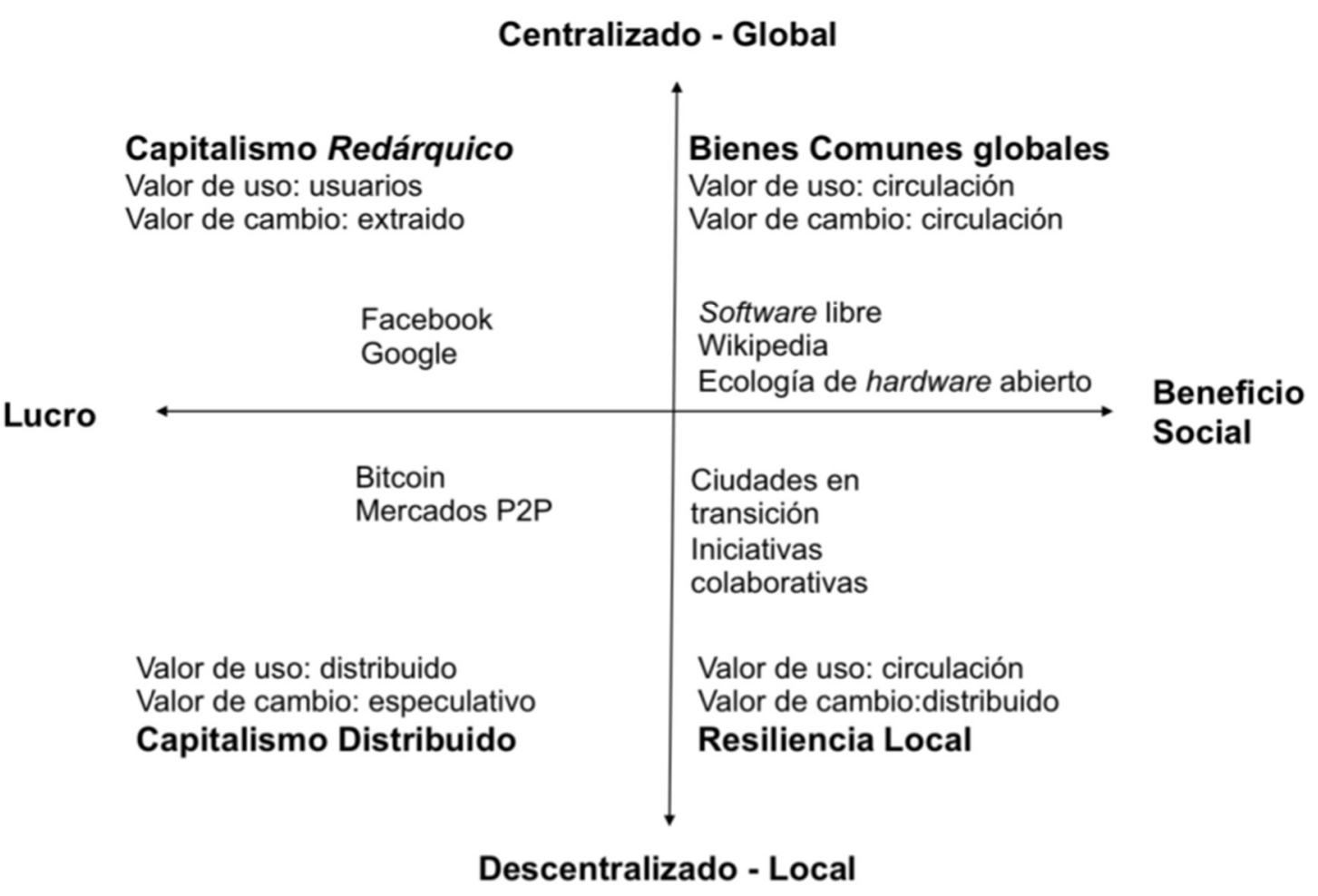

Fuente: traducido de Matties y Närhi (2016: 80)

En lo que se refiere a los contenidos de esta clasificación de escenarios, las tipologías refieren a los siguientes aspectos.

1. Capitalismo netárquico. El contenido (valor de uso) de las redes sociales (Facebook, Google, Twitter como ejemplos) es producido por los mismos usuarios. Se trata de un valor no retornado al productor (es extraído). El valor de cambio se crea a partir de los anunciantes y la venta de datos de los usuarios. El valor de cambio, por tanto, lo generan los capitalistas no por la propiedad intelectual y de los medios de producción, sino por el desarrollo y control de las plataformas participativas.

2. Capitalismo distribuido. Pequeños agentes consiguen ganar dinero extra mediante actividades económicas orientadas al alquiler, la prestación de algún servicio o la cesión temporal de algún objeto o su venta. La actividad de compartir hace que disminuya el coste de transacción. Frente a la idea de comunidad y de participación en la toma de decisiones sobre la 
configuración de la red, se parte del impedimento claro de que la propiedad de la red no es común. El valor de uso está distribuido en el conjunto de usuarios de la red (incorporan sus productos, trabajo, etc). El beneficio que obtienen (un dinero extra sobre la actividad principal de sustento, o en ocasiones, su única y muy limitada fuente de ingresos) contrasta con los grandes beneficios que obtienen los propietarios de la plataforma, y que se destinan a operaciones financieras globales con fines especulativos. La idea de red distribuida hace referencia la ausencia de un centro individual o colectivo. Los nodos de la red se vinculan unos a otros de modo que ninguno de ellos tiene poder de filtro sobre el contenido que se transmite en la red. Desaparece por tanto la divisoria entre centro y periferia característica de las redes centralizadas y descentralizadas. Las plataformas de economía colaborativa están produciendo una reestructuración del mercado laboral, fomentando el autoempleo. El riesgo de la fluctuación del mercado y del trabajo en sí se transfieren del empresario al trabajador.

3. Bienes comunes globales. En este caso, el principal aspecto es que se comparte información y conocimiento. La producción y la propiedad entre iguales da pie a la creación de un valor común, frente a la apropiación privada. Su principio es el conocimiento libre y el trabajo en equipo. Por ello, los ejemplos de esta creación de valor común tienen que ver con las iniciativas de conocimiento libre, tipo Wikipedia y el software libre, ampliándose también a la producción de hardware libre (compartiendo planos de proyectos de bienes de equipo). Todo lo creado se pone a disposición de la sociedad. En contraste con la criptomoneda Bitcoin del anterior modelo (capitalismo distribuido), tenemos el Faircoin, con similar código y funcionamiento al primero. Si Bitcoin obtiene su valor por oferta y demanda, Faircoin lo hace mediante el consenso. Desde el punto de vista medioambiental, Bitcoin utiliza gran cantidad de energía por los complejos cálculos de los datos de las trasacciones (el sistema blockchain), mientras Faircoin precisa cantidad mínima de energía para los mismos procesos porque utiliza un hardware "minimalista".

4. Redes locales resilientes. Una red descentralizada formada por nodos autogestionados de iniciativas locales de transición que tienen como base la idea de comunidad. Resilientes ante los desafíos medioambientales y las consecuencias desigualitarias del sistema capitalista. Desigualdades y precariedad crecientes por la extensión de la misma economía digital. Se trata de un capital distribuido, porque intenta reformular el concepto de dinero. El dinero es un medio de intercambio basado en la confianza entre aquellos que lo usan. En el momento que se puede mantener esta confianza entre los miembros se puede conseguir independencia del 
empleo (sustituido por el concepto de "actividad" para el individuo y la comunidad), del dinero oficial y de las instituciones financieras. Las experiencias de las monedas sociales y complementarias y la microfinanciación y el ahorro colaborativo, serían ejemplos.

En términos de innovación social y su vertiente política, nos resulta especialmente interesante el global commons, que interpretamos como un proceso de integración en red de redes resilientes del modelo "local resilience". El análisis conduce, en todo caso, a la realidad observable de un proceso acelerado a partir de la crisis financiera de 2008 en el que se impone el trabajo autónomo, inmaterial y la colaboración (con sus matices y diferente intensidad) sobre unos valores (aunque sólo sean slogans). Y mucho más importante, prácticas que difunden la capacidad de las personas para asociarse, aprovechar sus capacidades y estar preparados para llevar a cabo proyectos comunes sin fines de acumulación y sin dirección o tutela externa. Los efectos de esta multiplicación de prácticas sociales, a las que aludíamos al inicio de este artículo como proceso de innovación social, va impregnando el espacio sociocultural no sólo del consumo y los estilos de vida, sino también el relativo a la dimensión participativa de la democracia en el ámbito sociopolítico.

\section{CONCLUSIONES Y DEBATE}

Resulta evidente, en virtud a las propuestas consideradas y actualmente en debate, que la confusión sobre la economía colaborativa, su finalidad y dependencia de los desarrollos tecnológicos, están permitiendo que una herramienta eficaz de transformación social quede sepultada en las ambigüedades definitorias. Esta situación se agrava al ser absorbida en las dinámicas comerciales propias del capitalismo de consumo, donde, al igual que pasara en su momento con el ecologismo y otras alternativas, la noción de "colaboración" se convierte en un atributo más que caracteriza los productos, conservando toda la lógica de la generación de plusvalías. En ese sentido, el análisis de estos procesos emergentes constituye una tarea académica, pero también socialmente significativa.

Será el conocimiento de la auténtica naturaleza que define las relaciones insertas en dichas dinámicas de intercambio social, económico y simbólico, el que permitirá, primero una adecuada medición. y posteriormente una posibilidad de seguimiento y evolución de cambio. Tanto en términos de grupos sociales como de alternativas ideológicas y de relación social.

La propuesta de Matties y Närhi (2016) constituye un punto de partida relevante al que, evidentemente, deberán ser incluidas las dinámicas sociales e ideológicas que conforman alternativas reales de "economía colaborativa". El salto cualitativo entre los escenarios de "resiliencia local" a "bienes comunes globales", 
gracias a la vertebración organizacional que facilitan las nuevas tecnologías de la comunicación y la información, define el gran desafío operacional pero también teórico, al que se enfrentan las visiones alternativas al capitalismo de consumo y financiero globalizado.

\section{REFERENCIAS}

Alaminos, A. y Penalva, C. (2013). "España: de los impactos de la crisis a las movilizaciones de protesta", Ecuador Debate, 59, 93-118.

Alaminos, A., Penalva C, Doménech Y. (2014). “Reacciones comunitarias a la crisis económica y social en España", Revista Azarbe: Revista Internacional de Trabajo Social y Bienestar, 3, 47-53.

Algar, R. (2007). “Collaborative Consumption”, Leisure Report, April, 16-17.

Alonso, L.E. (2017). “Consumo colaborativo: las razones de un debate”, RES, 26 (1), 87-95.

Belk, R. (2010). "Sharing”, Journal of Consumer Research, 36 (5), 715-734.

Belk, R. (2014). "Sharing Versus Pseudo-Sharing in Web 2.0”, Anthropologist, 18(1), 7-23.

Böcker, L. and Meelen, T. (2017). "Sharing for people, planet or profit? Analysing motivations for intended sharing economy participation", Environmental Innovation and Societal Transitions, 23, 28-39.

Bostman, R. y Rogers, R. (2010). What's Mine is Yours: The Rise of Collaborative Consumption, Nueva York, Harper Collins.

Caballero, G. y Soto, D. (2013). “La nueva sociología económica y el nuevo institucionalismo en sociología: enfoques contemporáneos", en X. C. Arias y G. Caballero (eds.) Nuevo institucionalismo: gobernanza, economía y políticas públicas. Madrid, CIS, 131-156.

Chaves, R. y Monzón, J.L. (2018). “La economía social ante los paradigmas económicos emergentes: innovación social, economía colaborativa, economía circular, responsabilidad social empresarial, economía del bien común, empresa social y economía solidaria", Revista de Economía Pública, Social y Cooperativa, 93, 5-50, DOI: 10.7203/CIRIEC-E.93.12901.

Codagnone, C. y Martens, B. (2016). Scoping the Sharing Economy: Origins, Definitions, Impact and Regulatory Issues.,Institute for Prospective Technological Studies Digital Economy Working Paper 2016/01. JRC100369

CNMC (2016). Estudio sobre los nuevos modelos de prestación de servicios y la economía colaborativa. Resultados preliminares. E/CNMC/004/15. Madrid, CNMC.

Union Europea (2014) Coping with the crisis. Eurobarometer Qualitative study Aggregate Report, Brusseles, UE.

[http://ec.europa.eu/commfrontoffice/publicopinion/archives/quali/q1_6342_ crisis_en.pdf] 
Comisión Europea (2016). 356 final, Bruselas 2.6.2016, Comunicación de la Comisión al Parlamento Europeo, al Consejo al Comité Económico y Social europeo y al Comité de las Regiones titulada “Una Agenda Europea para la economía colaborativa".

Felber, C. (2015). La economía del bien común. Bilbao. Deusto Ediciones.

Gansky, L. (2010). The Mesh: Why the Future of Business is Sharing. New York, Portfolio/Penguin.

Hamari, J., Sjöklint, M. and Ukkonen, A. (2016). “The sharing economy: Why people participate in collaborative consumption", Journal of Association for Information Sciencie and Technology, 67, 2047-2059. Doi:10.1002/asi.23552.

Laín, B. (2017). “Algunas limitaciones en la comprensión de la economía colaborativa en sentido emancipador". Revista Española de Sociología ,26(1), 109-114. Doi: 10.22325/fes/res.2017.6.

Magrinyá, F., y Balanzó, R. de (2015). “Innovación social, innovación urbana y resiliencia desde una perspectiva crítica: el caso de la autoorganización en el espacio urbano de Barcelona", en J. Subirats, y Á. García Bernardos, Innovación social y políticas urbanas en España. Experiencias significativas en las grandes ciudades. Barcelona: Icaria Editorial. 59-93.

Matties, A-L. y Närhi, K. (2016). The ecosocial transition of societies. The contribution of social work and social policy, New York, Roudledge.

Möhlmann, M. (2015). “Collaborative consumption: determinants of satisfaction and the likelihood of using a sharing economy option again.", Journal of Consumer Behavior, 14, 193-207. Doi: 10.1002/cb.1512.

OCDE (2018). Perspectivas de la OCDE sobre la Economía Digital 2017, México, Asociación Mexicana de Internet, https://doi.org/10.1787/9789264302211-es.

Pais, I. y Provasi, G. (2015). “Sharing Economy: A Step towards the ReEmbeddedness of the Economy?", Stato e mercato, 3, 347-377.

Piñeiro, C., Suriñach, R., Fernández Casadevante, J.L. (2017). “Entre el mercado y la cooperación social. Luces y sombras de las prácticas de consumo colaborativo", RES, 26 (1) 97-108.

Pirni, A. Raffini, L (2015). “La empresa social en España y en Italia durante la crisis ¿un laboratorio de innovación económica y social?" Obets, Revista de ciencias sociales. 10 (1), 127-158.

Polanyi, K. (1976). "El sistema económico como proceso institucionalizado", en M. Godelier (comp.) Antropología y economía, Barcelona. Anagrama. 155-178.

Raffini, L. (2016). "L'economia della condivisione tra retoriche, ambiguità e lati oscuri. Riflessioni a partire dal caso Airbnb", La Rivista delle Politiche Sociali / Italian Journal of Social Policy, 1/2016, 129-149.

Santos, B. de S. (2014). Democracia al borde del caos:ensayo contra la autoflagelación, México. Siglo XXI.

Shor, J. (2016) "Debating the sharing economy", Journal of Self-Governance and Management Economics, 4 (3), 7-22.

Slee, T. (2016). Lo tuyo es mío. Contra la economía colaborativa. Madrid. Taurus. 
Union Europea (2016). Flash Eurobarometer 438 - March 2016 “The use of collaborative platforms" Report.

[http://ec.europa.eu/commfrontoffice/publicopinion/index.cfm/ResultDoc/down load/DocumentKy/72885]

Zubero, I. (coord.) (2014). "¿Qué sociedad saldrá de la actual crisis?, en VII Informe sobre exclusión y desarrollo social en España. Madrid. Fundación Foessa, Cap. 6.

http://www.foessa2014.es/informe/uploaded/capitulos/pdf/06_Capitulo_6.p df [consulta: 22/04/2017].

Zubero, I. (2015). "Innovación social: una propuesta para pensar las prácticas sociales en clave de transformación", en Joan Subirats y Ángela García Bernardos (eds.). Innovación social y políticas urbanas en España, Barcelona. Icaria. 13-41.

ANTONIO FRANCISCO ALAMINOS CHICA es catedrático de Sociología Matemática en la Universidad de Alicante. Sus líneas de investigación son los métodos y técnicas de investigación social comparada, los procesos de socialización y aculturación y las dinámicas de cambio social y político.

CLEMENTE PENALVA VERDÚ es profesor titular en la Universidad de Alicante e investigador del Instituto Interuniversitario de Desarrollo Social y Paz. Sus principales líneas de investigación son capital social y movilización social, redes de apoyo mutuo y redes para la acción política, y la dimensión económica de la confianza social. 